\title{
Skew loops and quadric surfaces
}

\author{
Mohammad Ghomi* and Bruce Solomon
}

\begin{abstract}
A skew loop is a closed curve without parallel tangent lines. We prove: The only complete surfaces in $\mathbf{R}^{3}$ with a point of positive curvature and no skew loops are the quadrics. In particular: Ellipsoids are the only closed surfaces without skew loops. Our efforts also yield results about skew loops on cylinders and positively curved surfaces.
\end{abstract}

Mathematics Subject Classification (2000). Primary 53A04, 53A05; Secondary 53C45, $52 \mathrm{~A} 15$.

Keywords. Tantrix, skew loop, ellipsoid, positive Gauss curvature.

\section{Introduction}

Here we study the relationship between surfaces in $\mathbf{R}^{3}$ and closed curves without parallel tangent lines. Examples of such curves, which we call skew loops, were first constructed by B. Segre in $1968[19]^{1}$ to disprove a conjecture of H. Steinhaus. Quite recently, Wu constructed skew loops in every knot class [27], and the first author has written down explicit examples on convex surfaces [10]. ${ }^{2}$ Despite this general failure of Steinhaus' conjecture, however, Segre noted that it does hold for loops that lie on ellipsoids, paraboloids, and certain symmetric cylinders. Here we add convex hyperboloids to Segre's list, show that certain asymmetric cylinders do admit skew loops, and use these facts to prove that the positively curved quadrics are actually characterized by the absence of skew loops:

Theorem 1.1. Let $M$ be a connected 2-manifold, and $F: M \rightarrow \mathbf{R}^{3}$ be a $C^{2}$ immersion. Suppose that $F$ has positive Gauss curvature at a point of $M$. Then the following are equivalent:

(1) $F(M)$ lies on a quadric surface.

(2) $F(M)$ contains no $C^{2}$ skew loops.

* The first author was partially supported by the NSF grant DMS-0204190.

${ }^{1}$ Porter gave an apparently independent construction in 1970 [17].

2 In [10] skew loops were used to solve the "shadow problem" formulated by H. Wente, which is related to the stability of constant mean curvature surfaces. 
In particular, if $F$ is a complete immersion and admits no $C^{2}$ skew loops, it is an embedding, and $M$ is simply connected.

Any loop on a right cylinder over an open planar curve has a pair of vertical tangent lines, and hence cannot be skew. So for purposes of the implication $2 \Rightarrow 1$ in Theorem 1.1 the assumption of positive curvature at one point is not superfluous. Moreover, since closed surfaces (compact 2-manifolds without boundary) always have such a point, Theorem 1.1 yields:

Corollary 1.2. Ellipsoids are the only closed $C^{2}$ surfaces immersed in $\mathbf{R}^{3}$ which admit no $C^{2}$ skew loops.

Characterizations of ellipsoids have a long and rich history [3, p. 151], [16], [13]. Most such theorems, however, are stated and proved within the class of convex bodies, where the surfaces are a priori embedded, and topologically spherical. Ours avoids both these restrictions.

We prove Theorem 1.1 by developing a sequence of intermediate results: In Section 2 we use regular homotopy to show that positively curved surfaces admit no skew figure-eights (Proposition 2.5). Applying this fact in Section 3, we then prove that convex quadrics have no skew loops. This involves a Lorentzian generalization, following [8] and [21], of Jacobi's Theorem on indicatrices that bisect the sphere [22, p. 407]. In Section 4 we prove our asymmetric "cylinder lemma" (Proposition 4.1): Any cylinder with a strictly convex asymmetric base contains a skew loop. We then exploit this fact in Section 5, using a stretching argument, to show that surfaces without skew loops have symmetric local cross sections. By a result of W. Blaschke, this property characterizes quadrics, and thus gives Theorem 1.1.

We conclude with three appendices. The first proves a result first stated by Segre, which gives a strong converse to the asymmetric cylinder lemma mentioned above, but still leaves the existence of skew loops on certain cylinders undetermined. We discuss this and other open problems in Appendix B, then conclude with a few historical notes in Appendix C.

\section{Preliminaries: skew loops and their tantrices}

A $C^{k}$ immersed loop is a $C^{k}$ mapping $\gamma: \mathbf{S}^{1} \simeq \mathbf{R} / 2 \pi \rightarrow \mathbf{R}^{3}$ with nowhere-vanishing velocity $\gamma^{\prime}$. We say $\gamma$ is skew iff $k \geq 1$ and

$$
\gamma^{\prime}(t) \times \gamma^{\prime}(s) \neq 0
$$

for all distinct $t, s \in \mathbf{R} / 2 \pi$. The tantrix of $\gamma$ is the mapping $\tau: \mathbf{S}^{1} \rightarrow \mathbf{S}^{2}$ given by $\tau(t):=\gamma^{\prime}(t) /\left\|\gamma^{\prime}(t)\right\|$.

Note 2.1. We will frequently use the following observations: (i) affine bijections of $\mathbf{R}^{3}$ map skew loops to skew loops, and (ii) $\gamma$ is skew iff $\tau\left(\mathbf{S}^{1}\right)$ is embedded and 
disjoint from its antipodal reflection, i.e., $\tau(t) \neq \pm \tau(s)$ for all distinct $t, s \in \mathbf{R} / 2 \pi$.

The curvature of a $C^{2}$ immersed loop is the speed of its tantrix $\left(\left\|\tau^{\prime}(t)\right\|\right)$. In Sections 3 and 5, we need to perturb skew loops while keeping them skew:

Lemma 2.2. $C^{2}$ skew loops with nonvanishing curvature form an open subset in the space of all $C^{2}$ immersed loops in $\mathbf{R}^{3}$, relative to the $C^{2}$ topology.

Proof. Let $\gamma$ be a $C^{2}$ skew loop with tantrix $\tau$ and nonvanishing curvature. Then $\tau$ is $C^{1}$ immersed. Suppose $\widetilde{\gamma}$ is a $C^{2}$ loop close to $\gamma$ in the sense of $C^{2}$ metric on $C^{2}$ loop space. Then $\widetilde{\gamma}$ has nonvanishing curvature as well, and therefore has a $C^{1}$ immersed tantrix $\widetilde{\tau}$. Further, $\widetilde{\tau}$ is close to $\tau$ in the $C^{1}$ metric. So $\widetilde{\tau}$ is embedded, because $\tau$ is embedded, and embeddings are open in $C^{1}$ immersed loop space [14, p. 37]. Finally, since $\tau$ avoids its antipodal image, it avoids some neighborhood of that image. So (by the triangle inequality) $\widetilde{\tau}$ avoids its antipodal image as well, and $\widetilde{\gamma}$ is skew.

Deformations of loops through immersions - regular homotopies - arise naturally for us since they continuously deform the tantrix of a loop as well. A basic theorem of H. Whitney [26] states that in $\mathbf{R}^{2} \simeq \mathbf{C}$, every loop is regularly homotopic to either the figure-eight

$$
\gamma_{0}\left(e^{i t}\right):=\cos t(1+\mathbf{i} \sin t),
$$

or to one of the degree- $k$ circle coverings given by

$$
\gamma_{k}\left(e^{i t}\right):=e^{i k t}, \quad k= \pm 1, \pm 2, \ldots
$$

On $\mathbf{S}^{2} \simeq \mathbf{C} \cup\{\infty\}$, however, S. Smale [20] showed that there are just two regular homotopy classes: that of the figure-eight $\gamma_{0}$, and of the equator $\gamma_{1}$. These facts lead to the following lemmas, useful both here and in Section 3.

Lemma 2.3. Every $C^{2}$ loop on $\mathbf{S}^{2}$ is regularly homotopic in $\mathbf{S}^{2}$ to its own tantrix.

Proof. The $C^{1}$ homotopy $h:[0, \pi / 2] \times I \rightarrow \mathbf{S}^{2}$ given by

$$
h(\theta, t):=\sigma(t) \cos \theta+\tau(t) \sin \theta
$$

deforms any immersed curve $\sigma$ into its tantrix $\tau:=\sigma^{\prime}$. To see that $h$ is regular, recall the spherical Frenet equation $\tau^{\prime}=-\sigma+\kappa_{g} \nu$ where $\kappa_{g}$ is the geodesic curvature of $\tau$, and $\nu:=\sigma \times \tau$. Setting $\sigma_{\theta}(t):=h(\theta, t)$, we compute

$$
\sigma_{\theta}^{\prime}(t)=\sigma^{\prime}(t) \cos \theta+\tau^{\prime}(t) \sin \theta=\tau(t) \cos \theta+\kappa_{g} \nu(t) \sin \theta-\sigma(t) \sin \theta .
$$

Since $\sigma, \tau$, and $\nu$ are orthonormal, $\sigma_{\theta}^{\prime} \neq 0$. So $\sigma_{\theta}$ is an immersion.

Lemma 2.3 implies that the tantrix of any $C^{2}$-immersed loop in $\mathbf{S}^{2}$ is immersed, a well-known fact $[11,18]$ that generalizes to loops on any positively curved surface: 
Lemma 2.4. The tantrix $\tau$ of any $C^{2}$-immersed curve $\sigma$ on a positively curved surface $M$ is immersed in $\mathbf{S}^{2}$.

Proof. Parametrize $\sigma$ by arclength, so that $\tau=\sigma^{\prime}$. The component of $\tau^{\prime}$ along a unit normal $\mathbf{n}$ on $M$ is then given by $\left(\tau^{\prime}\right)^{\perp}=\left(\sigma^{\prime \prime}\right)^{\perp}=\mathrm{k}\left(\sigma^{\prime}\right) \mathbf{n}$ where $\mathrm{k}$ denotes normal curvature. Since $M$ is positively curved, $\mathrm{k} \neq 0$. Hence $\tau^{\prime} \neq 0$.

A $C^{k}$ figure-eight $\alpha$ on a surface $M$ is any $C^{k}$ loop regularly homotopic to a loop $\beta$ in an open coordinate disc $\phi: U \rightarrow \mathbf{R}^{2}$, with $\phi \circ \beta=\gamma_{0}$ (the "standard" figure-eight above). Lemmas 2.3 and 2.4 yield:

Proposition 2.5. Let $f: M \rightarrow \mathbf{R}^{3}$ be a $C^{2}$-immersed, positively curved surface. Then the tantrix of any figure-eight on $M$ is again a figure-eight. In particular, $M$ admits no skew figure-eights.

Proof. By definition, any figure-eight $\alpha \subset M$ is regularly homotopic to a copy $\beta$ of our "standard" figure-eight $\gamma_{0}$ in a coordinate disc $U$. Lemma 2.4 then implies that the tantrix $\tau_{\alpha}$ of $\alpha$ is regularly homotopic to that of $\beta: \tau_{\alpha} \sim \tau_{\beta}$. It therefore suffices to show that $\tau_{\beta}$ is a figure-eight on $\mathbf{S}^{2}$.

After a regular homotopy of $\beta$ we may assume that $U$ is so small that $f(U)$ is a graph over one of its tangent planes. Then, after an affine transformation, $\beta$ lies in a coordinate disc $U \subset M$ with image $f(U)$ contained in the graph of a convex $C^{2}$ function $h_{0}: D^{2} \rightarrow \mathbf{R}$, where $D^{2} \subset \mathbf{R}^{2}$ is the open unit disc. We may then realize $\beta$ as a graph, $\beta_{0}$, over a figure-eight $\gamma: \mathbf{S}^{1} \rightarrow D^{2}$ :

$$
\beta_{0}(t)=\gamma(t)+h_{0}(\gamma(t)) \mathbf{k}
$$

where $\mathbf{k}:=(0,0,1)$. We may also assume (dilate further if necessary) that the eigenvalues of the Hessian $D^{2} h_{0}$ lie between 0 and 1 throughout $D$. Now express the southern hemisphere of $\mathbf{S}^{2}$ similarly as the graph of a function $h_{1}: D^{2} \rightarrow \mathbf{R}$. The eigenvalues of $D^{2} h_{1}$ are everywhere at least 1 , so the graphs of the functions

$$
h_{\epsilon}(x):=h_{0}(x)+\epsilon\left(h_{1}(x)-h_{0}(x)\right)
$$

give a deformation of $f(U)$ into $\mathbf{S}^{2}$ through positively curved surfaces. By Lemma 2.4, the tantrices of the figure-eights $\beta_{\epsilon}(t):=\gamma(t)+h_{\epsilon}(\gamma(t)) \mathbf{k}$ are all immersed. In particular, $\tau_{\beta} \sim \tau_{\beta_{1}}$. By Lemma 2.3, $\tau_{\beta_{1}} \sim \beta_{1}$. Thus $\tau_{\beta} \sim \beta_{1}$, which is a figure-eight on $\mathbf{S}^{2}$.

\section{Nonexistence of skew loops on quadrics}

The tantrix of a $C^{3}$ loop on $\mathbf{S}^{2}$, if embedded, bisects the sphere ([8], [21]). It follows that the tantrix of a $C^{3}$ loop on $\mathbf{S}^{2}$ crosses either itself or its antipodal image, and hence that $\mathbf{S}^{2}$ contains no $C^{3}$ skew loops. Segre observed that, by affine invariance, this fact extends to ellipsoids and elliptic paraboloids. Here we 
sharpen the argument in [21] to rule out $C^{2}$ skew loops on these same surfaces, ${ }^{3}$ and craft a Lorentzian version that includes the two-sheeted hyperboloids.

Note 3.1. Our methods in this section do not apply to curves that are only $C^{1}$. Further, we do not know whether $\mathbf{S}^{2}$ admits a skew loop which is $C^{1}$ but not $C^{2}$.

Let $Q$ denote the symmetric bilinear form on $\mathbf{R}^{3}$ characterized by

$$
Q(\mathbf{x}, \mathbf{x})=x^{2}+y^{2}-z^{2},
$$

for all $\mathbf{x}:=(x, y, z) \in \mathbf{R}^{3}$. The connected non-singular level sets of $Q(\mathbf{x}, \mathbf{x})$ are hyperboloids of revolution, each homothetic to one of the following:

$$
\begin{array}{ll}
\Sigma:=\left\{\mathbf{x} \in \mathbf{R}^{3}: Q(\mathbf{x}, \mathbf{x})=-1, z>0\right\} & \text { (hyperboloid of two sheets) } \\
\widetilde{\Sigma}:=\left\{\mathbf{x} \in \mathbf{R}^{3}: Q(\mathbf{x}, \mathbf{x})=+1\right\} & \text { (hyperboloid of one sheet). }
\end{array}
$$

Differentiating $Q$ along an $\operatorname{arc} \sigma$ immersed in either $\Sigma$ or $\widetilde{\Sigma}$ gives

$$
Q\left(\sigma^{\prime}, \sigma\right) \equiv 0 \text {. }
$$

Thus:

Lemma 3.2. Every point $p$ in $\Sigma$ or $\widetilde{\Sigma}$ is $Q$-normal to that surface at $p$.

Next, parametrize $\Sigma$ and $\widetilde{\Sigma}$ by $X: \mathbf{R} \times(0, \infty) \rightarrow \mathbf{R}^{3}$ and $\widetilde{X}: \mathbf{R} \times \mathbf{R} \rightarrow \mathbf{R}^{3}$ respectively as follows:

$$
\begin{aligned}
X(u, v) & :=(\cos (u) \sinh (v), \sin (u) \sinh (v), \cosh (v)), \\
\widetilde{X}(u, v) & :=(\cos (u) \cosh (v), \sin (u) \cosh (v), \sinh (v)) .
\end{aligned}
$$

Since $Q\left(X_{u}, X_{u}\right), Q\left(X_{v}, X_{v}\right)>0$, and $Q\left(X_{u}, X_{v}\right)=0, Q$ induces a Riemannian metric on $\Sigma .{ }^{4}$ So we may define the $Q$-tantrix of an immersed loop $\sigma$ on $\Sigma$ via

$$
\tau_{Q}(t):=\frac{\sigma^{\prime}(t)}{\sqrt{Q\left(\sigma^{\prime}(t), \sigma^{\prime}(t)\right)}} .
$$

Note 3.3. Since $Q\left(\tau_{Q}, \tau_{Q}\right)=+1$, the $Q$-tantrix of a loop on $\Sigma$ lies on $\widetilde{\Sigma}$. Further, $\tau_{Q}$ is the radial projection of the (standard) tantrix $\tau$ into $\widetilde{\Sigma}$. Therefore, much like $\tau$, the $Q$-tantrix of a skew loop on $\Sigma$ is embedded, and avoids its antipodal image.

In contrast to $\Sigma, \widetilde{\Sigma}$ inherits a Lorentzian structure from $Q$. Indeed, the vectors

$$
e^{+}:=\frac{\widetilde{X}_{u}}{\cosh (v)}, \quad e^{-}:=\widetilde{X}_{v}
$$

\footnotetext{
${ }^{3}$ The absence of $C^{2}$ skew loops on spheres was also established in 1971 by White [25].

${ }^{4}$ This is the well-known hyperbolic metric on $\Sigma$.
} 
form a global frame on $\widetilde{\Sigma}$, with

$$
Q\left(e^{+}, e^{+}\right)=+1, \quad Q\left(e^{-}, e^{-}\right)=-1, \quad \text { and } \quad Q\left(e^{+}, e^{-}\right)=0 .
$$

If we project out the $Q$-normal direction, the standard covariant derivative $D$ on $\mathbf{R}^{3}$ becomes a torsion-free, $Q$-preserving connection $\nabla$ on $\widetilde{\Sigma}$. Let $\omega$ denote the corresponding connection 1-form associated to our frame $\left\{e^{+}, e^{-}\right\}$by setting

$$
\omega(z):=Q\left(\nabla_{z} e^{+}, e^{-}\right), \quad \text { for all } z \in T \widetilde{\Sigma} .
$$

One may verify that in the local coordinates associated with $\widetilde{X}$,

$$
\omega=-\sinh (v) d u
$$

and that in conjunction with Lemma 3.2, differentiation of (3.4) yields

$$
\begin{aligned}
& \nabla_{z} e^{+}=-Q\left(\nabla_{z} e^{+}, e^{-}\right) e^{-}=-\omega(z) e^{-}, \\
& \nabla_{z} e^{-}=+Q\left(\nabla_{z} e^{-}, e^{+}\right) e^{+}=-\omega(z) e^{+} .
\end{aligned}
$$

Lemma 3.4. If a loop $\alpha$ in $\widetilde{\Sigma}$ is the $Q$-tantrix of a $C^{2}$ loop on $\Sigma$, then $\int_{\alpha} \omega=0$.

Proof. Suppose $\alpha=\tau_{Q}$, the $Q$-tantrix of an $\operatorname{arc} \sigma$ immersed in $\Sigma$. Since $\tau_{Q}$ is a multiple of $\sigma^{\prime}$, (3.2) implies that $Q\left(\tau_{Q}, \sigma\right) \equiv 0$. Lemma 3.2 then yields that $\sigma(t)$ is tangent to $\widetilde{\Sigma}$ at $\tau_{Q}(t)$. So we may expand $\sigma$ relative to the frame field given by (3.3). Since $Q(\sigma, \sigma) \equiv-1$, and $\sigma$ is $C^{2}$, this uniquely determines a function $\theta: \mathbf{S}^{1} \rightarrow \mathbf{R}$ such that

$$
\sigma(t)=\sinh \theta(t) e^{+}+\cosh \theta(t) e^{-} .
$$

Note that we evaluate the frame vectors here at $\tau_{Q}(t)$. Differentiating the above with respect to $t$, using (3.7), yields

$$
\nabla_{\tau_{Q}^{\prime}} \sigma=\left(\theta^{\prime}-\omega\left(\tau_{Q}^{\prime}\right)\right)\left(\cosh \theta e^{+}+\sinh \theta e^{-}\right) .
$$

On the other hand, by Lemma $3.2, \tau_{Q}(t)$ is $Q$-normal to $\widetilde{\Sigma}$ at $\tau_{Q}(t)$. So

$$
0=\left(\sqrt{Q\left(\sigma^{\prime}, \sigma^{\prime}\right)} \tau_{Q}\right)^{\top}=\left(\sigma^{\prime}\right)^{\top}=\left(D_{\tau_{Q}^{\prime}} \sigma\right)^{\top}=\nabla_{\tau_{Q}^{\prime}} \sigma,
$$

which yields that $\omega\left(\tau_{Q}^{\prime}\right) \equiv \theta^{\prime}$ along $\tau_{Q}$. But the integral of $\theta^{\prime}$ vanishes along $\tau_{Q}$, since $\theta$ is continuous and $\tau_{Q}$ is a loop. Hence $\int_{\tau_{Q}} \omega=0$.

We now have the tools we need to prove that positively curved quadrics admit no skew loops, and thereby establish half of our main theorem.

Proof of the implication $1 \Rightarrow 2$ of Theorem 1.1. There are 3 cases:

Case 1: Hyperboloids. Each nappe of a hyperboloid of two sheets is affinely isomorphic to the hyperboloid $\Sigma$ defined by (3.1). So it suffices to show that $\Sigma$ admits no $C^{2}$ skew loops. Suppose, toward a contradiction, that there exists a 
$C^{2}$ skew loop $\sigma: \mathbf{S}^{1} \rightarrow \Sigma$, with $Q$-tantrix $\tau_{Q}$. Since $\Sigma$ is diffeomorphic to a plane, and $\sigma$ may not be a figure-eight (Proposition 2.5), Whitney's theorem forces $\sigma$ to be regularly homotopic to a $k$-fold tracing $c_{k}$ of some horizontal circle, $k \neq 0$. The $Q$-tantrix of $c_{k}$ is then a $k$-fold tracing $\tau_{k}$ of the circle $z \equiv 0$ in $\widetilde{\Sigma}$, and since $\Sigma$ has positive curvature, the homotopy $\sigma \sim c_{k}$ induces a regular homotopy $\tau_{Q} \sim \tau_{k}$ (Lemma 2.4). By Note 3.3, $\tau_{Q}$ is embedded, and disjoint from its own antipodal image. The embeddedness forces $k=1$, and along with the antipodal disjointness, this means that $-\tau_{Q}\left(\mathbf{S}^{1}\right) \cup \tau_{Q}\left(\mathbf{S}^{1}\right)$ bounds an annular domain $\Omega \subset \widetilde{\Sigma}$ with $C^{1}$ boundary. Combining Stokes' Theorem with Lemma 3.4, we then get $\int_{\Omega} d \omega=\int_{\partial \Omega} \omega=0$. By (3.6), however, $d \omega=\cosh (v) d u d v$, a non-vanishing 2form. So the integral of $d \omega$ cannot vanish, and we have our contradiction.

Case 2: Ellipsoids. All ellipsoids are affinely equivalent, so we need only check the spherical case, which has been discussed by Segre [19] and White [25]. Alternatively, one can proceed as in Lemma 3.4, replacing $\sinh$ and cosh by sin and cos respectively. After suitably restricting their domains, the parametrizations $X$ and $\widetilde{X}$ for $\Sigma$ and $\widetilde{\Sigma}$ now become patches for $\mathbf{S}^{2}$. Arguing as in Lemma 3.4, one then shows that the tantrix of a loop on $\mathbf{S}^{2}$ must annihilate the integral of the corresponding connection form (which is now $-\sin v d u$ ). The final argument of Case 1 then goes over almost verbatim, because $d(\sin v d u)=\cos v d u d v$ gives the area form on $\mathbf{S}^{2}$, except at the poles, which we can avoid with a slight rotation.

Case 3: Paraboloids. By affine equivalence, it suffices to rule out skew loops on the graph $z=x^{2}+y^{2}$. One easily checks that this paraboloid, call it $P$, can be $C^{2}$-approximated arbitrarily well on any compact subset by an ellipsoid of the form

$$
x^{2}+y^{2}+\left(\frac{z}{2 r}-r\right)^{2}=r^{2}
$$

Further note that, since $P$ has positive curvature, any loop on $P$ has nonvanishing curvature. Thus it follows from Lemma 2.2 that for sufficiently large $r$, any skew loop on $P$ can be perturbed to form a skew loop on one of the ellipsoids defined by 3.8 above. Such a loop would contradict the result of Case 2 , so $P$ contains no skew loop.

\section{Asymmetric convex cylinders}

When a $C^{k}$ loop $\gamma: \mathbf{S}^{1} \rightarrow \mathbf{R}^{2}$ bounds a convex domain, we say $\Gamma:=\gamma\left(\mathbf{S}^{1}\right)$ is a $C^{k}$ oval. We say $\Gamma$ is (centrally) symmetric when reflection through a point leaves it invariant. Otherwise, it is asymmetric. We say $\Gamma$ is strictly convex if $\gamma$ is $C^{2}$ and its curvature never vanishes. Our main aim in this section is to show:

Proposition 4.1 (Cylinder Lemma). The cylinder over any asymmetric, strictly convex $C^{2}$ oval $\Gamma \subset \mathbf{R}^{2}$ contains a $C^{2}$ skew loop with nonvanishing curvature. 
This follows easily once we prove three preliminary results. Our strategy boils down to the careful analysis of a classical parametrization: Recall that when $\Gamma$ is strictly convex, its outward unit normal $n: \Gamma \rightarrow \mathbf{S}^{1}$ is injective. We may therefore employ the support parametrization $\gamma: \mathbf{R} \rightarrow \mathbf{R}^{2}$ of $\Gamma$, given by

$$
\gamma(t):=n^{-1}\left(e^{i t}\right) \text {. }
$$

Note that one loses a derivative in passing from $\Gamma$ to $\gamma$. When $\Gamma$ is merely $C^{2}$, this somewhat complicates the proof that $\gamma$ is an immersion:

Lemma 4.2. Let $\Gamma \subset \mathbf{R}^{2}$ be a strictly convex $C^{2}$ oval, with support parametrization $\gamma$. Then $v:=\left\|\gamma^{\prime}\right\| \neq 0$. Moreover, $\Gamma$ is symmetric if and only if $v$ is $\pi$-periodic.

Proof. Define the support function of $\Gamma$ via

$$
h(t):=\left\langle e^{i t}, \gamma(t)\right\rangle
$$

(real inner product). Since $\left\{e^{i t}, i e^{i t}\right\}$ is a basis for $\mathbf{R}^{2}$, we then have a $2 \pi$-periodic $C^{1}$ function $\mu: \mathbf{R} \rightarrow \mathbf{R}$ such that

$$
\gamma(t)=(h(t)+i \mu(t)) e^{i t} .
$$

By (4.1), $e^{i t}$ is normal to $\Gamma$ at $\gamma(t)$, so we also have

$$
\gamma^{\prime}(t)=v(t) i e^{i t}
$$

Now differentiate (4.3) and compare with (4.4) to see that $\mu=h^{\prime}$ and

$$
\gamma(t)=\left(h(t)+i h^{\prime}(t)\right) e^{i t} .
$$

As $\gamma$ is $C^{1}$, this shows that $h$ is $C^{2}$. Further, differentiating (4.5) and using (4.4), we get

$$
v=h^{\prime \prime}+h
$$

We now make indirect use of the curvature formula $\kappa:=\left\langle\gamma^{\prime \prime}, i \gamma^{\prime}\right\rangle /\left\|\gamma^{\prime}\right\|^{3}$ to show that $v \neq 0$. If $\gamma$ is $C^{2}$, one can differentiate (4.4) to evaluate $\gamma^{\prime \prime}$, and directly calculate $\kappa=1 / v$. Since $\Gamma$ is strictly convex, we have $\kappa \neq 0$, and hence $v \neq 0$, as claimed. Here $\gamma$ is only $C^{1}$, so we first approximate $\Gamma$ in $C^{2}\left(\mathbf{S}^{1}, \mathbf{R}^{2}\right)$ by a sequence of $C^{3}$ ovals $\Gamma_{\ell}$. The support parametrization of each $\Gamma_{\ell}$ will then be $C^{2}$, so that for $\Gamma_{\ell}$, we do have $\kappa_{\ell}=1 / v_{\ell}$. But the curvatures $\kappa_{\ell}$ and speeds $v_{\ell}$ of the $\Gamma_{\ell}$ 's converge uniformly to $\kappa$ and $v$ respectively. In the limit, we therefore obtain $\kappa=1 / v$ as claimed.

To get our final conclusion, suppose that $\Gamma$ is symmetric about the origin. The reflection $\rho(x)=-x$ then sends the tangent line at $\gamma(t)$ to some parallel line tangent to $\Gamma$. Given (4.1), the only such tangency occurs at $\gamma(t+\pi)$. Thus

$$
\gamma(t+\pi)=-\gamma(t)
$$

for all $t \in \mathbf{R}$. By (4.2) this forces both $h$ and $v=h^{\prime \prime}+h$ to be $\pi$-periodic. 
Conversely, suppose $v$ is $\pi$-periodic. Then all its odd Fourier coefficients must vanish. Since $h^{\prime \prime}+h=v$, the same must hold for $h$, modulo a solution $\left\langle w, e^{i t}\right\rangle$ of the homogeneous equation $h^{\prime \prime}+h=0$. By (4.2), however, we eliminate this anomaly if we translate $\Gamma$ by $-w$. Doing so makes $h \pi$-periodic, and by virtue of (4.5), the oval parametrized by $\gamma$ is now $\rho$-invariant. The original (untranslated) oval $\Gamma$ is then symmetric.

We shall define and denote the even and odd parts of a function $f: \mathbf{S}^{1} \rightarrow \mathbf{R}$ by

$$
f_{+}(t):=\frac{f(t)+f(t+\pi)}{2}, \quad \text { and } \quad f_{-}(t):=\frac{f(t)-f(t+\pi)}{2},
$$

respectively, identifying $\mathbf{S}^{1}$ with $\mathbf{R} / 2 \pi$ via $e^{i t} \leftrightarrow t$. With this notation, we can give a simple condition for the skewness of a "graphical" loop $\widetilde{\gamma}$ on the cylinder over $\Gamma$ :

Lemma 4.3. Suppose $\Gamma \subset \mathbf{R}^{2}$ is a strictly convex $C^{2}$ oval with support parametrization $\gamma$. Let $z: \mathbf{S}^{1} \rightarrow \mathbf{R}$ be $C^{1}$, and set $v:=\left\|\gamma^{\prime}\right\|, \mathbf{k}:=(0,0,1)$. Then $\widetilde{\gamma}(t):=\gamma(t)+z(t) \mathbf{k}$ is a skew loop if and only if for all $t \in \mathbf{R}$, we have

$$
v_{+}(t) z_{+}^{\prime}(t)-v_{-}(t) z_{-}^{\prime}(t) \neq 0 \text {. }
$$

Further, if $z$ is $C^{2}$, then $\widetilde{\gamma}$ has nonvanishing curvature.

Proof. Expressing $\gamma^{\prime}$ as in (4.4) above, and using the identity $i e^{i \tau} \times \mathbf{k}=e^{i \tau}$, we compute that

$$
\begin{aligned}
\widetilde{\gamma}^{\prime}(t) \times \widetilde{\gamma}^{\prime}(s) & =\left(\gamma^{\prime}(t) \times \gamma^{\prime}(s)\right)+\left(z^{\prime}(s) \gamma^{\prime}(t)-z^{\prime}(t) \gamma^{\prime}(s)\right) \times \mathbf{k} \\
& =v(t) v(s) e^{i t} \times e^{i s}+v(t) z^{\prime}(s) e^{i t}-v(s) z^{\prime}(t) e^{i s} \\
& =v(t) v(s) \sin (t-s) \mathbf{k}+v(t) z^{\prime}(s) e^{i t}-v(s) z^{\prime}(t) e^{i s} .
\end{aligned}
$$

Note that $\widetilde{\gamma}$ fails to be skew whenever this quantity vanishes for some $t, s \in \mathbf{R}$, with $t \not \equiv s \bmod 2 \pi$. Since the $\mathbf{k}$ component vanishes only when $s \equiv t+\pi \bmod 2 \pi$, $\widetilde{\gamma}$ is thus skew if and only if

$$
v(t) z^{\prime}(t+\pi)+v(t+\pi) z^{\prime}(t) \neq 0
$$

for all $t \in \mathbf{R}$. Now note that for any function $f: \mathbf{R} / 2 \pi \rightarrow \mathbf{R}$, we have the identities

$$
\begin{aligned}
f(t) & =f_{+}(t)+f_{-}(t), & & f_{+}(t+\pi)=f_{+}(t), \\
f(t+\pi) & =f_{+}(t)-f_{-}(t), & & f_{-}(t+\pi)=-f_{-}(t) .
\end{aligned}
$$

Applying these to $v$ and $z^{\prime}$ in the preceding formula gives the first conclusion of the lemma. Finally, note that, since $\gamma$ is strictly convex, $\left\|\gamma^{\prime \prime}(t)\right\| \neq 0$. Thus, if $z$ is $C^{2}$, then $\left\|\widetilde{\gamma}^{\prime \prime}(t)\right\| \neq 0$ as well. So $\widetilde{\gamma}$ has nonvanishing curvature.

The technical result below will provide the key constructive step in our proof of the cylinder lemma. 
Lemma 4.4. Let e, o: $\mathbf{S}^{1} \rightarrow \mathbf{R}$ be continuous functions which are even and odd respectively, and suppose that $e+o>0$. Then either $o \equiv 0$, or we have a continuous function $\mu: \mathbf{S}^{1} \rightarrow \mathbf{R}$, such that:
(1) $\int_{\mathbf{S}^{1}} \mu=0$,
(2) $\mu$ is even, and
(3) $e \mu>-o^{2}$.

Proof. Assume $o \not \equiv 0$, and identify $\mathbf{S}^{1}$ with $\mathbf{R} / 2 \pi$ as usual. To prove the lemma we will construct a continuous function $\mu:[0, \pi] \rightarrow \mathbf{R}$ with
$\left(1^{\prime}\right) \int_{0}^{\pi} \mu(t) d t=0$
$\left(2^{\prime}\right) \mu(\pi)=\mu(0), \quad$ and
$\left(3^{\prime}\right) e \mu>-o^{2}$ on $[0, \pi]$.

The even extension of this function to all of $\mathbf{S}^{1}$ then clearly has the properties (1), (2), and (3) that we seek.

To begin, observe that our hypotheses automatically imply $e>0$ throughout $\mathbf{S}^{1}$. Otherwise, the evenness of $e$ would imply $e \leq 0$ at both points of some antipodal pair $t,-t \in \mathbf{S}^{1}$. Since we assume $e+o>0$ everywhere, this would force $o>0$ at both $t$ and $-t$, contradicting the oddness of $o$. We thus have positivity of $e$, which allows us to define

$$
\tau:=\frac{1}{\pi} \int_{0}^{\pi}\left(\frac{o(t)^{2}}{1+e(t)}\right) d t>0 .
$$

Next, note that the zero set of an odd function is both nonempty, and invariant under reflection through the origin. After a rotation, we may therefore assume $o(0)=o(\pi)=0$, and define the function we seek:

$$
\mu(t):=\tau-\frac{o^{2}(t)}{1+e(t)} .
$$

Clearly, $\mu$ satisfies $\left(1^{\prime}\right)$. And we arranged that $o(0)=o(\pi)=0$, so we have $\mu(0)=\mu(\pi)=\tau$, which gives $\left(2^{\prime}\right)$. Finally, we obtain $\left(3^{\prime}\right)$ by combining our definition of $\mu$ with the positivity of $e$ and $\tau$ :

$$
e(t) \mu=e(t) \tau-\left(\frac{e(t)}{1+e(t)}\right) o(t)^{2}>-\left(\frac{e(t)}{1+e(t)}\right) o(t)^{2}>-o(t)^{2} .
$$

This proves the Lemma.

We now prove the main result of this section, our cylinder lemma:

Proof of Proposition 4.1. By Lemma 4.3, it suffices to produce a height function $z: \mathbf{S}^{1} \rightarrow \mathbf{R}$ such that, for all $t \in \mathbf{R}$,

$$
v_{+}(t) z_{+}^{\prime}(t)>v_{-}(t) z_{-}^{\prime}(t)
$$

where $v$ is the speed of the support parametrization of $\Gamma$. First, note that our asymmetry hypothesis on $\Gamma$ combines with Lemma 4.2 to guaranteed that $v$ is not even, and hence $v_{-} \not \equiv 0$. Moreover, being odd, $v_{-}$has a well-defined antiderivative on $\mathbf{S}^{1}$. We form $z_{-}$by taking any such antiderivative and subtracting off its 
average on $\mathbf{S}^{1}$. Clearly, this makes $z_{-}$a (non-trivial) odd function, and because $v_{-}$is continuous, $z_{-}$is $C^{1}$. Since $z_{-}^{\prime}=-v_{-}$, (4.6) now becomes

$$
v_{+}(t) z_{+}^{\prime}(t)>-\left(v_{-}(t)\right)^{2} \text {. }
$$

It remains to construct an even $C^{1}$ function $z_{+}: \mathbf{S}^{1} \rightarrow \mathbf{R}$ whose derivative satisfies (4.7). Lemma 4.4 does precisely that: Set $e:=v_{+}, o:=v_{-} \not \equiv 0$ there, and let $z_{+}^{\prime}:=\mu$. Lemma 4.2 ensures us that $e+o=v_{+}+v_{-}=v>0$, so Lemma 4.4 indeed applies. Conclusions (1) and (2) of the latter now guarantee that $z_{+}^{\prime}$ has an even antiderivative $z_{+}$on $\mathbf{S}^{1}$, and conclusion (3) reduces to the key estimate (4.7).

\section{Quadricity of surfaces without skew loops}

Our first step in this section is to use the existence of skew loops on asymmetric convex cylinders (Proposition 4.1) to restrict the symmetry of surfaces without skew loops:

Lemma 5.1. Let $S \subset \mathbf{R}^{3}$ be a $C^{2}$ embedded surface without skew loops. Suppose that there exists a plane $H \subset \mathbf{R}^{3}$ which meets the interior of $S$ transversely along a strictly convex oval $\Gamma:=S \cap H$. Then $\Gamma$ is symmetric.

Proof. After a rigid motion we may assume that $H$ coincides with the $x y$-plane. Since $S$ meets $H$ transversely along $\Gamma$, we may choose $\epsilon>0$ small enough to make

$$
S^{\prime}:=\{(x, y, z) \in S:|z|<\epsilon\}
$$

a topological annulus transversal to $H$ with $\partial S^{\prime} \cap H=\emptyset$. Let $C$ denote the cylinder perpendicular to $H$ with base $\Gamma$. Then $S^{\prime}$ may be represented as a graph over $C$. That is, there exists an open neighborhood $A$ of $\Gamma$ in $C$ and a $C^{2}$ function $g: A \rightarrow \mathbf{R}$ such that $S^{\prime}=\{a+g(a) \nu(a): a \in A\}$, where $\nu$ is the outward unit normal vector field on $S$. Now use the dilatations $\mu_{c}: \mathbf{R}^{3} \rightarrow \mathbf{R}^{3}$, defined for each $c \geq 1$ by $\mu_{c}(x, y, z):=(x, y, c z)$, to define a 1 -parameter family of $C^{2}$ functions

$$
g_{c}: A \rightarrow \mathbf{R}, \quad g_{c}:=g \circ \mu_{1 / c} .
$$

Note that $g_{c}$ and its derivatives tend to zero uniformly on $A$ as $c \rightarrow \infty$. This follows from the continuity of $g$ and the chain rule, because $g=0$ on $\Gamma$, while near $\Gamma$, the derivatives of $g$ are continuous because $S^{\prime}$ intersects $H$ transversally.

Suppose now that $\Gamma$ is not symmetric. Then Proposition 4.1 gives a $C^{2}$ skew loop $\gamma: \mathbf{S}^{1} \rightarrow C$ with nonvanishing curvature. After a (shrinking) dilatation, we may assume that $\gamma\left(\mathbf{S}^{1}\right) \subset A$. For every $c \geq 1$, we may then define a loop $\gamma_{c}$ on the affinely stretched surface $\mu_{c}\left(S^{\prime}\right)$ by setting

$$
\gamma_{c}(t):=\gamma(t)+g_{c}(\gamma(t)) \nu(\gamma(t)) .
$$


Since $g_{c} \rightarrow 0$ uniformly on $\gamma\left(\mathbf{S}^{1}\right)$ along with its derivatives as $c \rightarrow \infty$, we see that $\gamma_{c} \rightarrow \gamma$ in the $C^{2}$ sense. It then follows, by Lemma 2.2 , that $\gamma_{c}$ eventually becomes skew. Thus, for sufficiently large $c>0$, the stretched surface $\mu_{c}\left(S^{\prime}\right)$ admits a skew loop. As an affine map, however, $\mu_{c}$ sends skew loops to skew loops. So $S^{\prime}$ must itself admit a skew loop - a contradiction.

By a convex body $K \subset \mathbf{R}^{3}$ we mean a compact convex subset with nonempty interior. We say planes $P_{1}, P_{2}$ are close if we can represent them by linear equations $\left\langle n_{1}, x\right\rangle=h_{1}$ and $\left\langle n_{2}, x\right\rangle=h_{2}$, with $\left|n_{1}-n_{2}\right|^{2}+\left|h_{1}-h_{2}\right|^{2}<\epsilon$ for some $\epsilon>0$.

Theorem 5.2 (Blaschke [2]). Let $K \subset \mathbf{R}^{3}$ be a convex body, whose boundary is $C^{2}$ near a point $p \in \partial K$. Suppose that whenever a plane sufficiently close to $T_{p} \partial K$ intersects $K$, its intersection with $\partial K$ is centrally symmetric. Then a neighborhood of $p$ in $\partial K$ lies on a quadric surface.

Blaschke's result localizes a theorem of Brunn that characterizes ellipsoids as convex bodies having only symmetric cross sections. ${ }^{5}$ Coupling it to Lemma 5.1, we quickly complete the proof our main theorem.

Proof of the implication $2 \Rightarrow 1$ of Theorem 1.1. Let $X \subset M$ be the largest open subset whose image $F(X)$ lies on a quadric. Then $X$ is also closed, and $M$ is connected, so we need only show that $X \neq \emptyset$. To do so, let $U$ be an open neighborhood of a point $p$ in $M$ where the curvature is positive. We may choose $U$ small enough so that $S:=F(U)$ is the graph of a function on the tangent plane $T_{F(p)} \partial K$. Since the curvature is positive at $p$, this function has positive definite Hessian and is therefore convex. So $S$ lies on the boundary of a convex body $K \subset \mathbf{R}^{3}$. Since $S$ has positive curvature at $F(p)$, the tangent plane $T_{F(p)} \partial K$ intersects $K$ only at $F(p)$. This gives an $\epsilon>0$ so that every plane $H \subset \mathbf{R}^{3}$ within distance $\epsilon$ of $T_{F(p)} \partial K$ satisfies $H \cap \partial S=\emptyset$. Then $\Gamma:=H \cap \partial K$ lies in $S$. Whenever the intersection is transversal $\Gamma$ is a $C^{2}$ strictly convex oval, because $S$ has positive curvature. Lemma 5.1 now makes $\Gamma$ symmetric. But $\Gamma$ was an arbitrary transverse cross-section of $S$ near $p$, so Blaschke's Theorem 5.2 forces a neighborhood of $p$ to lie on a quadric surface. This completes the proof.

\section{Appendix A. Symmetric cylinders}

Proposition A.2 below gives a strong converse to the existence of skew loops on asymmetric cylinders (Proposition 4.1). This result was known to Segre [19], but we recount a proof for completeness. Let us agree that an $L$-periodic unit-speed

\footnotetext{
${ }^{5}$ Olovjanischnikoff (see [15] and [4, p. 346]) proves an even more general version requiring no regularity at $p$.
} 
loop $c: \mathbf{R} \rightarrow \mathbf{R}^{2}$ has arclength symmetry with respect to a point $p \in \mathbf{R}^{2}$ if $c(t+L / 2)=p-c(t)$ for all $t \in \mathbf{R}$.

Note A.1. For embedded loops, one can show that arclength symmetry is equivalent to central symmetry. In particular, Proposition A.2 holds for cylinders over embedded centrally symmetric loops. For immersed loops, arclength symmetry is slightly stronger than central symmetry, however; centrally symmetric figureeights, for instance, admit no arclength-symmetric parametrization. Indeed, one can put a skew loop on the cylinder over a centrally symmetric figure-eight. Example: $\widetilde{\gamma}(t)=\left(\cos t, \sin 2 t, \frac{t}{\pi}-\left(\frac{t}{\pi}\right)^{15}\right)$. The arclength symmetry condition below therefore seems essential.

Proposition A.2. If a $C^{1}$ loop $\Gamma \subset \mathbf{R}^{2}$ admits a parametrization with arclength symmetry, then the cylinder $S:=\Gamma \times \mathbf{R} \subset \mathbf{R}^{3}$ admits no skew loops which are transverse to the lines in $S$.

Proof. Suppose $\Gamma$ has length $L$, and has an $L$-periodic parametrization $c: \mathbf{R} \rightarrow \mathbf{R}^{2}$ which is arclength-symmetric about the origin. Let $S:=\Gamma \times \mathbf{R}$, and suppose $\widetilde{\gamma}: \mathbf{S}^{1} \rightarrow S$ is a $C^{1}$ loop. We may then reparametrize $\widetilde{\gamma}$ via

$$
\widetilde{\gamma}(t):=c(t)+z(t) \mathbf{k},
$$

where $z$ is $C^{1}$ and $n L$-periodic for some $n \in \mathbf{Z}$. By our symmetry assumption, $c(t+L / 2)=-c(t)$. Hence

$$
c^{\prime}(t+L / 2)=-c^{\prime}(t)
$$

and

$$
\int_{0}^{n L}\left(z^{\prime}(t)+z^{\prime}(t+L / 2)\right) d t=\left.(z(t)+z(t+L / 2))\right|_{0} ^{n L}=0 .
$$

The mean value theorem for integrals now gives a $t_{0} \in[0, n L]$ for which

$$
z^{\prime}\left(t_{0}+L / 2\right)=-z^{\prime}\left(t_{0}\right) .
$$

Equations (A.1), (A.2) and (A.3) therefore combine to yield $\widetilde{\gamma}^{\prime}\left(t_{0}+L / 2\right)=-\widetilde{\gamma}^{\prime}\left(t_{0}\right)$. This makes the tangent lines to $\widetilde{\gamma}$ at $t_{0}$ and $t_{0}+L / 2$ parallel, a contradiction.

\section{Appendix B. Open problems}

Which surfaces in $\mathbf{R}^{3}$ admit skew loops? Theorem 1.1 settles this question for surfaces with a point of positive curvature, so it remains to ask:

Problem B.1. Which nonpositively curved surfaces admit skew loops?

Could it be true that the only surfaces without skew loops and a point of negative curvature are quadric, mirroring Theorem 1.1? If so, it would remain to 
study flat surfaces. ${ }^{6}$ Complete flat surfaces are generalized cylinders [12]. When embedded and symmetric, these admit no skew loops, which are transversal to the generators of the cylinder, by Proposition A.2. The main open question about flat surfaces is then:

Problem B.2. Which asymmetric cylinders admit skew loops?

Proposition 4.1, shows that strict convexity is sufficient, and one can show that the tantrix of any loop on a cylinder whose base has winding number $|\iota|>1$ must self-intersect; these cylinders do not admit skew loops.

Our work raises some regularity questions too. We state one of them in Note 3.1: Does $\mathbf{S}^{2}$ contain a $C^{1}$ skew loop? The regularity of the underlying surface raises another: Skew loops necessarily have one derivative, so a version of Theorem 1.1 in the $C^{1}$ category would be fairly optimal with regard to regularity. Our last question highlights a simple relevant test case:

Problem B.3. Does a cylinder capped by hemispheres admit skew loops?

This surface is $C^{1}$ and piecewise quadric; if it admits a skew loop, Theorem 1.1 is already optimal.

Finally, we remark that when one regards $\mathbf{R}^{3}$ as $\mathbf{R} \mathbf{P}^{3}$ minus a plane at infinity, all ellipsoids are projectively equivalent, not just to each other, but to the elliptic paraboloids and 2-sheeted hyperboloids too. The referee has observed that these are precisely the quadrics on which we have ruled out skewloops, and hence our results may extend to $\mathbf{R P}^{\mathbf{3}}$ in an interesting way. We hope to explore this possibility in a future paper.

\section{Appendix C. Historical notes}

According to P. Du Val [7], H. Steinhaus conjectured the non-existence of skew loops in 1966 during a lecture given at Sussex. B. Segre, present at this lecture, responded by proposing a counterexample in a lecture of his own the next day. Segre eventually published a corrected version of his counterexample in 1968 [19]. Porter's version of the construction in 1970 [17] is somewhat more explicit, but Segre's paper contains many other results, including the non-existence of skew loops on spheres. To prove the latter fact, he appeals to a "bel teorema" published by W. Fenchel in 1934 [8]: The tantrix of a spherical curve, if embedded, bisects $\mathbf{S}^{2}$. It seems that this result was absorbed by very few beside Segre. It immediately implies a well-known theorem of Jacobi on the normal indicatrix of a space curve,

\footnotetext{
6 Serge Tabachnikov has recently ruled out skew loops on negatively curved quadrics, and on simply-connected flat surfaces [23]. In fact, by extending the technique of White [25], he rules out $n$-dimensional compact skew "branes" on all hyperquadrics in $\mathbf{R}^{n+2}$ for all $n$.
} 
but the many subsequent references to Jacobi's Theorem we know (e.g. [22], [5], [6], and even Fenchel's own 1951 survey [9]!) make no mention of it. It has since been rediscovered at least twice: by Avakumovic [1], and by the second author [21].

The non-existence of skew loops on spheres was also proved by J. H. White [25] in 1971 using a Morse-theoretic argument. Unlike Segre, who notes that the result extends to ellipsoids and elliptic paraboloids, White mentions only the sphere. Neither author suggests that hyperboloids admit no skew loops, nor gives any hint that they surmised our main result here.

Acknowledgements. We thank Ralph Howard, Richard Montgomery, and Serge Tabachnikov for their interest and helpful comments. We also thank the University of California at Santa Cruz and Stanford University for facilitating the early stages of our collaboration.

\section{References}

[1] V. Avokumović, Über geschlossene Kurven auf der Kugel, Srpska Akad. Nauka. Zbornik Radova Matematicki Inst. 7 (1) (1951), 101-108 (Serbo-Croatian, German summary).

[2] W. Blaschke, Gesammelte Werke, Band 4, Affine Differentialgeometrie. Differentialgeometrie der Kreis- und Kugelgruppen, edited by Burau, S. S. Chern, K. Leichtweiß, H. R. Müller, L. A. Santaló, U. Simon and K. Strubecker, Thales-Verlag, Essen, 1985.

[3] T. Bonnesen and W. Fenchel, Theory of convex bodies, translated from the German and edited by L. Boron, C. Christenson and B. Smith, BCS Associates, Moscow, Idaho, 1987.

[4] G. R. Burton. Some characterisations of the ellipsoid, Israel J. Math. 28 (4) (1977), 339-349.

[5] S. S. Chern. Studies in Global Geometry and Analysis, MAA Studies in Mathematics, Vol. 4. Mathematical Assoc. of America (printed by Prentice-Hall), 1967.

[6] M. P. DoCarmo, Differential Geometry of Curves and Surfaces, Prentice Hall, Englewood Cliffs, New Jersey, 1976.

[7] P. Du Val, Beniamino Segre. Bull. London Math. Soc. 11 (1979), 215-235.

[8] W. Fenchel, Über einen Jacobischen Satz der Kurventheorie, Tôhoku Math J. 39 (1934), 95-97.

[9] W. Fenchel, On the differential geometry of closed space curves, Bull. Amer. Math. Soc. $\mathbf{5 7}$ (1951), 44-53.

[10] M. Ghomi, Shadows and convexity of surfaces, Ann. of Math. 155 (2002), 281-293.

[11] H. Gluck and L.-H. Pan, Embedding and knotting of positive curvature surfaces in 3-space, Topology 37 (4) (1998), 851-873.

[12] P. Hartman and L. Nirenberg, On spherical image maps whose Jacobians do not change sign, Amer. J. Math. 81 (1959), 901-920.

[13] E. Heil and H. Martini, Special convex bodies, in: Handbook of convex geometry, Vol. A, B, 347-385, North-Holland, Amsterdam, 1993.

[14] M. W. Hirsch, Differential topology, Springer-Verlag, New York, 1994.

[15] S. Olovjanischnikoff, Ueber eine kennzeichnende Eigenschaft des Ellipsoides, Uchenye Zapiski Leningrad State Univ., Math. Ser. 83 (12) (1941), 114-128.

[16] C. M. Petty, Ellipsoids, in: Convexity and its applications, 264-276, Birkhäuser, Basel, 1983. 
[17] J. R. Porter, A note on regular closed curves in $E^{3}$, Bull. Acad. Polon. Sci. Sér. Sci. Math. Astronom. Phys. 18 (1970), 209-212.

[18] H. Rosenberg, Hypersurfaces of constant curvature in space forms, Bull. Sci. Math. 117 (2) (1993), 211-239.

[19] B. Segre, Sulle coppie di tangenti fra ioro parallele relative ad una curve chuisa sghemba, Hommage au Professeur Lucien Godeaux, 141-167, Libraire Universitaire, Louvain, 1968.

[20] S. Smale, Regular curves on Riemannian manifolds, Trans. Amer. Math. Soc. 87 (1958), $492-512$.

[21] B. Solomon, Tantrices of spherical curves, Amer. Math. Monthly 103 (1) (1996), 30-39.

[22] M. Spivak, A Comprehensive Introduction to Differential Geometry, Vol. 3, Publish or Perish, Inc., Boston, 1975.

[23] S. Tabachnikov, On skew loops, skew branes, and quadratic hypersurfaces, Preprint.

[24] J. L. Weiner, Flat tori in $S^{3}$ and their Gauss maps, Proc. London Math. Soc. (3) 62 (1) (1991), 54-76.

[25] J. H. White, Global properties of immersions into Euclidean spheres, Indiana Univ. Math. J. 20 (1971), 1187-1194.

[26] H. Whitney, On regular closed curves in the plane, Compositio Math. 4 (1937), 276-284.

[27] Y.-Q. Wu, Knots and links without parallel tangents, to appear in Bull. London Math. Soc.

Mohammad Ghomi

University of South Carolina

Department of Mathematics

Columbia, SC 29208

USA

e-mail: ghomi@math.sc.edu

URL: www.math.sc.edu/ ghomi
Bruce Solomon

Indiana University

Department of Mathematics

Bloomington, IN 47405

USA

e-mail: solomon@indiana.edu

URL: php.indiana.edu/ solomon

(Received: January 7, 2002)

To access this journal online: http://www.birkhauser.ch 